\title{
Time Development of new Hydrogen Transmission Pipeline Networks for France
}

\author{
Jean ANDRE ${ }^{1}$, Stéphane AURAY ${ }^{2}$, Daniel DE WOLF ${ }^{3}$, \\ Mohamed-Mahmoud MEMMAH ${ }^{4}$, and Antoine SIMONNET ${ }^{5}$ \\ ${ }^{1}$ GDF Suez, R\&D Division, Paris, France \\ ${ }^{2}$ CREST-Ensai, EQUIPPE (EA 4476), Université du Littoral, \\ Campus de Ker-Lann, Rue Blaise Pascal, BP 37203, 35172 Bruz \\ cedex, France, Email : stephane.auray@ensai.fr \\ ${ }^{3}$ TVES (EA 4477), Université du Littoral, Dunkerque, 189B avenue \\ Maurice Schumann, BP 5526, 59379 Dunkerque, France, Email: \\ daniel.dewolf@univ-littoral.fr \\ ${ }^{4}$ INRA, UR1115 Plantes et Systèmes de culture Horticoles, \\ Domaine St. Paul, Site Agroparc, Avignon, F-84914 France, \\ ${ }^{5}$ TOTAL Raffinage Marketing, Paris, France
}

October 15, 2014

\begin{abstract}
The development of an hydrogen economy will need a transportation infrastructure to deliver hydrogen from production sites to end users. For the specific case of hydrogen, pipelines networks compete with other hydrogen carriers: compressed gas trucks and liquid cryogenic trucks. In this paper, we deal with the determination of the temporal deployment of a new hydrogen transportation infrastructure. Starting from the expected final horizon pipelines network, we propose a backward heuristic approach. The proposed approach is illustrated on a French regional hydrogen transportation tacking into account two scenarios for hydrogen penetration into the fuel markets. We showed that for the mid term perspective and low market share, the trucks are the most economical options. However, for the long term, the pipeline option is considered as a economical viable option as soon as the hydrogen energy market share for the car fueling market reaches $10 \%$.
\end{abstract}

Keywords: Hydrogen, energy economics, time scheduling, network deployment, penetration rates.

\section{Introduction}

Hydrogen is today considered by the actors of the energy sector as future transportation fuel reconciling energy security and sustainable development constraints 
(See Doe [10] as North American reference, Castello et al.[8] or HyFrance [11] for European references). However, the development of the hydrogen energy is facing many social, technological, and economic barriers. The lack of major transport infrastructure is often pointed out as one of these major barriers (See Johnson et al. [12]). This infrastructure has to ensure the hydrogen delivery from production sites to the end-users. Although the transmission/distribution pipelines will constitute the main part of this infrastructure in the long term vision, alternative transportation modes have to be considered in the short term. Alternative transportation modes could be more competitive compared to the pipelines when the hydrogen energy has a lower market share. Therefore, the spatial and temporal deployment of the hydrogen transportation networks should be carefully studied and all modes should be taken into account by the decision-makers. To achieve a better understanding of these aspects, economic evaluation of such networks and their environmental impacts (carbon capture) are main criteria to support the decision-makers. Many national and international research projects have been funded especially in Europe (See Castello et al. [8], Ball et al. [2] and André et al. [1]) and the USA (See Johnson and Ogden [12]).

Thus, many studies have been conducted to investigate all these issues and a literature review shows that the spatial aspect of the hydrogen infrastructure was more studied than the the temporal deployment. Indeed, the deployment problem belongs to the large class of multistage network design problem which are widely used in the telecommunication sector (See Triadou [24], Kubat [15], Yi [29], Olorunniwo and Jensen [19]). We review the most interesting work dealing with the spatial and temporal deployment of oil and gas transmission and distribution networks in order to position our work in its context. Yi [29] solves the expansion time problem with the help of linear integer programming on single source tree networks. On gunbarrel structures ${ }^{1}$ with pipes and compressor stations, Olorunniwo and Jensen [19] showed that for an infinite horizon of time, the time of the optimum expansion of the pipeline segments and compressor stations can be determined independently of the pipe sizes and compressor capacity expansions. They also showed that the time of expansion for each facility can be determined independently of the other facilities. In fact, they apply a decomposition approach where they change the complex multi-facility capacity expansion problem into a series of much simpler single facility problems.

Similarly, in the Carbon Capture and Storage literature, Middleton and Bielicki [17] develop SimCSS, a scalable infrastructure model, for the design of pipelines network connecting carbon sources and storage reservoirs. The authors formulate the problem as a mixed integer linear program (MILP) and use ILOG's CPLEX 11.0 mixed integer optimizer to solve it. Note that the SimCSS model doesn't take into consideration the nonlinear relations between pipe diameters and gas flows. It also assumes that the objective function is linear by considering only fixed construction costs and this study was dealing only with spatial aspect of hydrogen network transmission/distribution.

In the recent Hydrogen Energy literature, Baufume et al. [6] consider the design of both transmission pipeline network across all the districts of Germany and distribution networks to supply refueling stations within the districts based on

\footnotetext{
${ }^{1}$ Main pipeline with gas flowing in one direction compressed with multiple serial compressor stations.
} 
minimal spanning trees. The main feature of the transmission model is the selection of pre-defined preferred routes along side the existing natural gas networks. The sizing of the network diameters as well as compression costs were taken into account and the results for the transmission network are very similar to the sizes found on the French case with a large majority of diameters ranging from $100 \mathrm{~mm}$ up to $300 \mathrm{~mm}$ [1]. However, these models simulate the final state of the network at the 2050 horizon and do not explore the temporal network deployment. Besides, they do not consider the competition of neither the alternate delivery modes by trucks nor the on-site hydrogen productions especially at the early stage of the hydrogen market share.

In UK, Balta-Ozkan and Baldwin [3] consider the problem of the spatial development of hydrogen economy in a low-carbon UK energy system. Authors embedded a spatial hydrogen module within the UK MARKAL (MARKet ALlocation) energy system model. They characterize the UK hydrogen network around 6 supply facilities and 12 demand centers. As delivery modes, three choices were considered in this study namely: liquid delivery by tankers, gaseous pipeline networks, and small-scale on-site production.This study considers the competitiveness of hydrogen supply by pipes compared to others modes and takes into account the temporal aspect through illustrative scenarios

Finally, in the US, Johnson and Ogden [12] evaluate the cost and design of various infrastructure deployment strategies. They develop a network optimization tool for identifying the lowest cost centralized production and pipeline transmission infrastructure within real geographic regions in the southwestern United States. For this purpose, they used the Hydrogen Production and Transmission (HyPAT) model for optimizing a long-term hydrogen pipeline. The HyPAT model takes into account the location and the diameters of pipelines, the location and size of plants and their actual production capacities. The problem is formulated as a MILP in the General Algebraic Modeling System (GAMS). To solve this problem, the authors used the CPLEX software. Authors considered five market penetration levels and run the optimization process for each one. In each step, the model started from the infrastructure built at a previous market penetration level considered as constraint.

The goal of all these approaches is to delay at the latest stage the required investment to match the progressive levels of demand (the preference for paying later is represented with the use of a discount factor on future costs). To solve this problem, two main approaches could be used: the dynamic programming and heuristics. The dynamic programming approach (see Bertsekas [4]) is very suitable to solve problems where we have an underlying discrete-time dynamic system and a cost function that is additive over time. Otherwise, heuristic approaches are necessary to address the multistage problem. The basic ideas of these heuristics is to solve the one-period problem stage by stage without taking into account the impact on the other period. Even if our approach is based on the same principle, the specificity of our approach lies in the fact that the pipelines is compared with other hydrogen carriers namely the compressed gas trucks and the liquid cryogenic trucks. We will thus consider at each time period three states for each nodes corresponding to the different supply mode. It means that the number of states can be a large number regarding the number of nodes of the network. Moreover, the transitions between a state delivery by pipeline for one 
node at one period to the following period might be difficult to compute since the network optimal topology (and the associated diameter) for one time step might be strongly different from the optimal topology to the following time steps with an overall increased demand. The use of the dynamic programming approach has not been considered as a practical approach for these two main reasons.

The paper is organized as follows. Section 2 presents the ECOTRANSHY model and states the time deployment problem. Section 3 presents the problem formulation including the computation of the different costs corresponding to the alternative transportation modes. Section 4 is dedicated to the presentation of the proposed backward heuristic. Section 5 applies this heuristic on a practical application: the development of a new hydrogen transport infrastructure for the North of France. We present also in section 5 the mains observations concerning the time development of this new pipelines infrastructure. Finally, Section 6 presents some conclusions and perspectives for future works.

\section{Hydrogen supply chains: model and options}

In this section, we describe ECOTRANSHY model used in this study before giving some elements on competitive supply chains options considered in this paper.

\subsection{The ECOTRANSHY model}

In the framework of PAN-H French national program for hydrogen, the ECOTRANSHY project aimed to develop an economic model for the spatial and the temporal deployment of hydrogen transmission network for France. A software called "ECOTRANSHY" was developed for these purposes. This software allows answering the following questions:

- What is the optimal design (topology) and dimensioning (diameters) of a new hydrogen pipelines transmission network?

- What is the impact of the geographical aggregation (country wide, region wide, and city scales) on this optimal solution?

- Taking into account the progressive hydrogen market share increase, when becomes the pipeline network economically viable versus the trucks delivery?

- What will be the expansion planning for an pipeline infrastructure with decentralized regional sources?

Based on the state of the art in that field, we developed a reliable and transparent network cost model taking into account hydrogen technical constraints. The software architecture is presented in Figure 1.

This original model takes into consideration transmission and distribution networks characteristics, operating conditions, energy and material costs. The developed software is composed by the following main subroutines: 


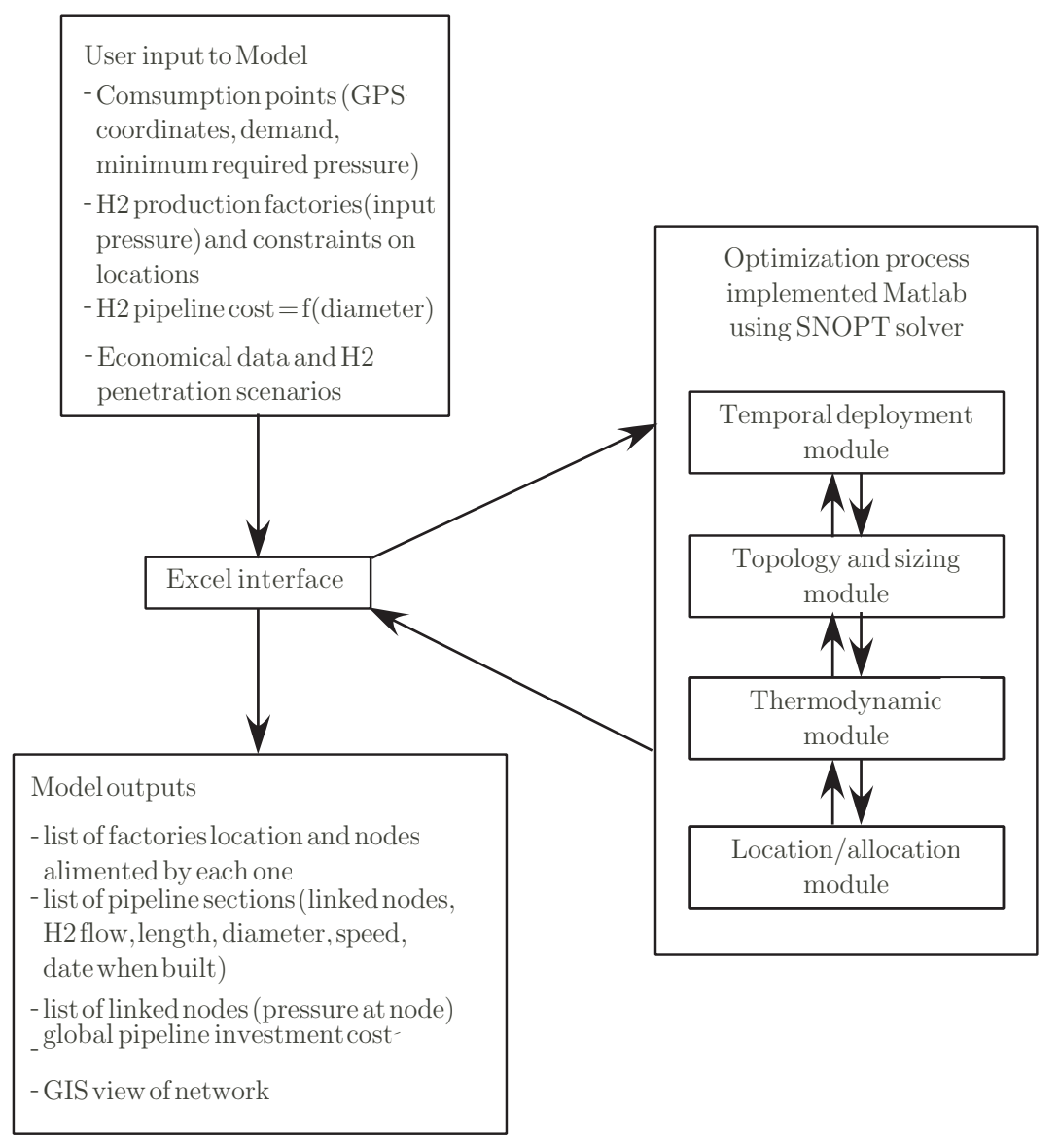

Figure 1: Software architecture 
- an input subroutine that allows the user to enter all the data concerning the consumption nodes, the hydrogen production, the costs and the Hydrogen market shares;

- an optimization subroutine which computes the network topology and the pipes sizing, the location of the Hydrogen production centers and the allocation of demand points to this center, and finally, the temporal deployment of the network taking into account the thermodynamic specific equations for Hydrogen,

- an output subroutine which gives the factory location, the network, the flows, the pressures and the global investment cost.

In a previous work (See André et al. [1]), we presented the design problem (the network topology) and the pipe diameter sizing module. We thus addressed the problem of the optimal design and dimensioning of an hydrogen transmission network. This design problem includes the topology determination and the pipelines dimensioning problem. Using a local search method, we simultaneously look for the least cost topology of the network and for the optimal diameters of the pipelines.

The application to the case of development of future hydrogen pipeline networks in France has been conducted at the local, regional and national levels. The present paper considers the hydrogen transition options to assess the feasibility of these options and to answer the last two questions. Beyond the network design and sizing, we investigate the time deployment of the pipeline network.

\subsection{Hydrogen Competing Supply Chains options}

As a matter of fact, even if the choice of the pipeline may make sense over the long-term with enough high throughputs in the pipes and enough long distances, the choice of the pipeline can be challenged at lower levels of demand by the truck delivery. At early stages of development of the hydrogen supply chain, the delivery by trucks brings the combined advantages of low initial investment costs and the flexibility to deliver small and infrequent amounts of hydrogen. However, the supply chains by trucks have to rely on significant investments on liquefiers (for the cryogenic option) or compressor stations (to fill the trailers) with long term payback periods. Then, based on market share evolutions, the pipeline CAPEX and OPEX costs will be compared at each decision time steps to the corresponding costs on the truck side to determine the time to switch from the trucks to the pipeline option. In this competition between transportation modes, the tradeoff have to be found as soon as the economy of scale provided by the pipeline with enough high rates compensates for the flexibility given by the truck deliveries.

This time phasing is usually critical to spread over time the investment but in our case its benefits can be assessed with regards to alternate transportation modes. In case of an emerging hydrogen economy for the car fueling business, the hydrogen from the production sources to the refueling stations may be carried through different more flexible modes than the pipeline mode. As the pipeline 
requires a significant amount of capital costs with a long term return on investments (ROI), the trucks propose a viable alternate choice to deliver small and infrequent amounts of hydrogen with limited investments (especially in the early phase of development).

At the upstream of the supply chains, we will assume large scale hydrogen production units to supply the whole nation with in between one to four production sites for France. The technologies that could fit such requirements are either fossil fuel based (Steam Methane Reformers) or renewable energy based (Wind or High Temperature electrolyzers). The choices and the costs associated to these production technologies are not discussed in this paper. Besides, let us note that the small on site production facilities close to the refueling stations are not considered in this approach as well. The only information regarding the centralized production are that the hydrogen will be under gaseous form and that the hydrogen pressure right outside the production facility will be around 15bars (usually observed outside of a Pressure Swing Adsorption unit).

At the downstream end of the supply chain, we will consider hydrogen cars with compressed gaseous hydrogen tanks. Many storage technologies can accommodate hydrogen at very high pressures. However, in this paper, we won't discuss the different alternatives and associated costs. The only information is that the storage pressure will be in between 400 and 700 bars (based on actual existing technologies) to store enough energy content and reaching an high enough cars mileage autonomy.

In between these 2 ends of the hydrogen economy, the delivery system can be decomposed into 2 parts : transportation parts from the production sites to the cities gates and distribution parts from the cities gates to a network of refueling stations. As in the previous paper from the authors André et al. [1], the pipeline solution can be implemented with the same optimal methodology at both scales with transportation pipeline systems on one hand and distribution pipeline systems on the other hand. The connection at the boundaries of the transportation system are set to 100 bars at the injection and 35 bars at the city gates in order to have the gas flowing over long distance with only compression at the plant level and then neither on-line re-compression on the transportation system nor at the cities gates to reach few bars at the refueling stations. Let us note that in this paper unlike the previous paper, we will consider for the pipeline options the full costs including not only the pipeline investment but also the compression investment at the plant level and at the refueling stations in order to be consistent with alternate transportation modes.

The delivery by trucks option will be decomposed in the same way as the pipeline with primary flows with large trucks from production sites to storage facilities at cities gates either with large liquid bulk storage or with warehouses hosting high pressure trailers depending on the selected options. The secondary flows of smaller trucks will connect these storage facilities to the network of refueling stations. Although a part of the investments related to the tractors and the drivers won't be impacted by the hydrogen's state, a significant part of these investments (trailers and the upstream and downstream equipments) will depend on the phase of hydrogen: gaseous or liquid:

- In the gaseous case, unlike for the pipeline option which requires medium 
compression power at the central plant (injection around 100 bars) but high booster compression rates at the refueling stations (injection between 450 and 700 bars in the car's tank), the gas trailer will require significant amount of compression at the central plant to feed the trailer (at up to 400 bars) but limited compression on the refueling site will be necessary (only for tanks higher than 400 bars).

- In the liquid case, the main additional investment consists of the liquefaction unit located right after the central plant. Besides, the liquid hydrogen will need dedicated cryogenic tanks both at the production site and at the delivery station as well as on the truck with cryogenic trailers. Since liquid tanks inside the cars are too costly, delivery to cars will be under gaseous form. Hence, vaporization will occur and booster compression will be necessary to reach the desired pressure level in the gaseous cars tanks.

A summary of the different delivery options and their associated features is given in the figure 2 .

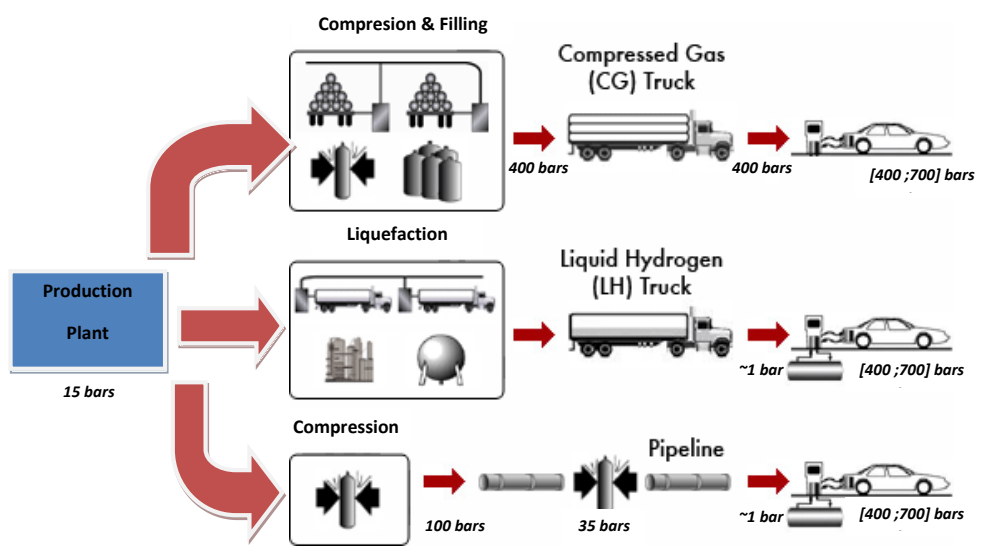

Figure 2: Hydrogen Supply Chains, source : adapted from US DOE H2A, [10]

\section{Problem formulation}

From the elements given in in the previous section, we propose to decompose the overall costs of the 3 transportation options considered in this paper as follows:

- for the pipeline option, the costs include the centralized compression at the hydrogen production source costs, the transportation costs, and the 
on-site compression costs. Note that no on-line re-compression will be considered from the injection source and the network exit points (as in André et al. [1]).

- for the gaseous truck option, costs are decomposed into the central compression costs to feed the gas into the trailers (compressed at 500 bars), transportation costs, and on site compression costs.

- for the liquid truck option, costs include the liquefaction costs, the transportation costs and one site compression costs. In fact, at the delivery points, the liquid is vaporized at ambient temperature and pressures (few bars). Therefore, an on-site compression is necessary to reach the storage requirements.

First of all, we give the mathematical formulation of each building block constituting each of the three transportation options: compression, pipeline, liquefaction and logistic costs. Then, we explain the proposed economic criterion allowing us to compare the three hydrogen transportation modes.

\subsection{Compression and liquefaction costs}

At both ends of the supply chains, compression will be necessary to transport and deliver hydrogen over long distances. On the upstream end, at the production source, a medium-low pressure is available around 15 bars. On the downstream end, at the exit points, on site booster compression will be needed to match the high pressure requirement of the static hydrogen tanks at the refueling stations (usually at 415 bars).

For the three transportation options considered in-between these two ends, compressions costs will need to be included and addressed for each of the solutions. The compression power, noted $P$ in $\mathrm{KW}$, either at the central plant or at the delivery site is computed as follows (see general references in the books for gases of Mohitpour [18] and Katz [14] and also in report dedicated to hydrogen [8]):

$$
P=\frac{Q}{3600 * 24 * 33.33} * \frac{Z T R}{M_{H_{2}} \eta_{\text {comp }}} * \frac{N \gamma}{\gamma-1} *\left(\left(\frac{P_{\text {out }}}{P_{\text {in }}}\right)^{\frac{\gamma-1}{N \gamma}}-1\right)
$$

with

- $Q$ the flow rate (in $\mathrm{kWH}$ per day) by taking a volumic high heating value (HHV) of 2.99 cubic meter $/ \mathrm{kWh}$ specific to hydrogen gas,

- $P_{i n}$ the inlet pressure of the compressor (suction),

- $P_{\text {out }}$ the outlet pressure of the compressor (discharge),

- $Z$ the hydrogen compressibility factor equal to 1 as an approximation,

- $N$ the number of compressor stages,

- $T$ the inlet temperature of the compressor $(278 \mathrm{~K})$,

- $\gamma$ the diatomic constant factor (1.4),

- the molecular mass of hydrogen $(2.15 \mathrm{~g} / \mathrm{mol})$, 
- $\eta_{\text {comp }}$ the compressor efficiency ratio (here taken as $75 \%$ ),

- the universal constant of ideal gas $R=8.314 \mathrm{JK}^{-1} \mathrm{~mol}^{-1}$,

For the compression investment costs used at the connection points of the pipeline and for the truck transportation chain, we consider the following linear relation with the compression power (adapted from the National Research Council [26] for on-site compression with no investment fixed cost) with $P$ compression power in $\mathrm{KW}$ :

$$
C A P E X_{\text {compression }}=2545 * P[\$]
$$

In addition to the CAPEX, we consider a yearly maintenance fee of $3 \%$ of the CAPEX and the electricity costs to run the compressor station:

$$
O P E X_{\text {compression }}=(0.85 \times 8760 \times e / D T E) * P[\$]
$$

with $e$, the electricity costs $(60 € / \mathrm{MWh}$ in 2010) and DTE, the Driver Thermal Efficiency (90\%).

Concerning the liquefaction, we consider one liquefier located at the central plant for the cryogenic option. The capital costs of this liquefier have been inferred from existing ones in Europe. The corresponding costs are roughly of $1.1 € / \mathrm{kg}$.

\subsection{Pipeline costs}

For the pipeline option, we made the following assumptions: the injection pressures are set to 60 bars and the exit pressures to 40 bars.

As the hydrogen flows through the pipeline system, the pipe sizing of the diameter $D_{i j}$ will depend on the pressure drop equation linking the square pressures to the flowrate $Q_{i j}$ and the diameter (See De Wolf and Smeers [9]):

$$
Q_{i j}=K\left(D_{i j}\right) \sqrt{p_{i}^{2}-p_{j}^{2}}
$$

In a previous paper from the authors [1], the authors devise a methodology to optimally design both the size and the topology of the network to meet the injection and delivery pressures.

The capital expenditures for the pipeline investments per unit of length is a quadratic function of the pipe diameter (see Parker [20]) with $D$ denoting the pipe diameter (in $\mathrm{mm}$ ):

$$
C A P E X_{\text {pipe }}=418869+762.8 * D+2.306 * D^{2}[\$ / k m]
$$

In addition to the CAPEX, we apply a yearly operation and maintenance fee of $2 \%$ of the CAPEX. 


\subsection{Logistic costs to transport hydrogen by trucks}

Let us define the truck depots as the warehouse where tractors are assigned in garages and where drivers will start their shifts. Some assumptions are made on the truck supply chain in order to simplify the cost calculation to deliver each customer or each intermediate standalone storage (both called delivery nodes). We first consider that truck depots will be based at the production plants which limit the driving time from the plant to a depot. Second, the nodes will only be delivered by full drop trucks which means that a truck will start from the plant, deliver the full amount of product within the trailer and come back to plant without delivering to any nearby customers. Third, we will consider that a truck will be fully assigned to this delivery node to compute the average yearly cost of a full time truck to refill the considered node. As a consequence, no trucks are shared among several delivery nodes and the investment is justified just for delivering one specific delivery node. Although this voluntary restriction can make sense for delivering an intermediate storage at the city gate, it will hardly hold for delivering small refueling stations. Then, this cost will be readjusted in proportion to the ratio between the yearly demand for a given year over the total quantity transported by this assigned truck per year.

Based on the aforementioned assumptions, the first component of the cost structure for logistics will be mainly based on an estimation of time extended over the year. Then, if we consider $L$ (in $\mathrm{km}$ ) as the distance between the central production plant, one round trip will take this amount of time time (in hours) :

$$
\text { time }=2 \cdot \frac{L}{S_{t}}+L T+U T
$$

with $S_{t}$ the speed of the truck $(\mathrm{km} / \mathrm{h}), L T$, the loading time at the central plant and $U T$ the unloading time at the refuelling station. Then, in one year $(365 \times$ 24 hours $=8760$ hours), the maximal number of round trips is the following:

$$
N_{r t}=\frac{8760 \times \text { TruckAvailability }}{\text { time }} .
$$

with the TruckAvailability as a percentage of time of the year. And the maximum hydrogen quantity that can be carried with this truck:

$$
Q M A X_{y}=K_{\text {trailer }} \cdot N_{r t}
$$

with $K_{\text {trailer }}$ the maximal capacity of a trailer.

The $O P E X_{T r}$ associated to the truck operations are then computed as the sum of the following terms:

- The yearly cost of diesel is:

$$
\text { Diesel }_{\text {costtotal }}=\text { dieselcost } * \text { truckConsumption } * 2 * L * N_{r t}
$$

- The yearly cost of labor is:

$$
\text { LaborCosttotal }=\text { Laborcosthour } * \text { time } * N_{r t}
$$


- The yearly costs of insurance, licenses, maintenance associated to the truck is :

$$
\text { OperationCosttotal }=2 * L * O \& M \operatorname{cost} * N_{r t}
$$

The cost data such as the loading time, the unloading time, the truck availability, the truck capacities, the labor cost, the CAPEX for gaseous and cryogenic trailer, their operations and maintenance cost and their lifetime are given in table 1 .

\begin{tabular}{|c|c|c|}
\hline & Variable & Value \\
\hline Average Truck speed & $S_{t}$ & $40 \mathrm{~km} / \mathrm{h}$ \\
Loading Time for cryogenic trailers (hours) & $\mathrm{LT}$ & 3 \\
Unloading Time for cryogenic trailers (hours) & $\mathrm{UT}$ & 3.5 \\
Truck availability $(\%)$ & TruckAvailabilty & 80 \\
Cryogenic Trailer Capacity $(\mathrm{kg})$ & $K_{\text {trailer }}^{\text {cryo }}$ & 4000 \\
Gaseous Trailer Capacity $(\mathrm{kg})$ & $K_{\text {trailer }}^{\text {gas }}$ & 650 \\
Truck fuel consumption $(\mathrm{L} / \mathrm{km})$ & truckConsumption & 0.39 \\
Tractor Fuel costs $(€ / \mathrm{L})$ & dieselcost & 1.1 \\
Labor Costs for Drivers $(€ / \mathrm{h})$ & Laborcosthour & 20 \\
CAPEX gaseous trailer $(€)$ & $C A P E X_{\text {trailer }}^{\text {gas }}$ & 350000 \\
CAPEX cryogenic trailer $(€)$ & $C A P E X_{\text {trailer }}^{\text {cryo }}$ & 625000 \\
CAPEX tractor $(€)$ & $C A P E X_{\text {tractor }}$ & 100000 \\
Operations \& Maintenance costs $(€ / \mathrm{km})$ & OPEX $X_{\text {Tr }}$ & 0.047 \\
Tractor Lifetime (years) & tractorlifetime & 5 \\
Trailer Lifetime (years) & trailerlifetime & 20 \\
\hline
\end{tabular}

Table 1: Truck Supply Chains: Technical specific values and cost assumptions. Sources: US Department of Energy H2A Delivery Analyses [10].

\subsection{Economic criterion: equivalent annual costs}

Based on the classical economic literature, the problem of comparing investments with unequal lives can come either from the traditional net present value (NPV) method or from the equivalent annual cost (EAC) method. Net present value and equivalent annual cost are two discounted cash flow criteria for comparing investment proposals but the annual cost method appears in the engineering literature and has not appeared in the accounting literature (see Jones and Smith [13]). As we position this paper in the engineering literature, the economic criterion is based on the computation of $C_{m}$ the average yearly cost of the equipment. Let us consider an asset (truck, pipe,...) with a initial capital expenditure (CAPEX), an yearly operational expenditure (OPEX) and a lifetime of $n$ years. If $i$ is considered as the discount rate, then the total sum of costs summing immediate investment with foreseen discounted fixed yearly operational costs over the lifespan at year 0 can be computed as follows:

$$
\sum_{t=1}^{n} \frac{C_{m}}{(1+i)^{t}}=C A P E X+\sum_{t=1}^{n} \frac{O P E X}{(1+i)^{t}}
$$


We can deduce from the following formula the average equivalent annual cost of the equipment:

$$
C_{m}=\frac{C A P E X}{\sum_{t=1}^{n} \frac{1}{(1+i)^{t}}}+O P E X=\frac{C A P E X}{\left[\frac{1-(1+i)^{-n}}{i}\right]}+O P E X
$$

The unit for $C_{m}$ is $€$ /year. For the last equality, let us recall that the sum of $n$ first terms of a geometric series of a common ratio of $r=1 /(1+i)$, is

$$
\sum_{t=1}^{n} \frac{1}{(1+i)^{t}}=\left[\frac{1-(1+i)^{-n}}{i}\right]
$$

This equivalent annual cost will be computed for each piece of the different considered supply chain. Then each cost will be added to be able to compare the 3 supply chains between them. Let us note that it is not necessary to divide this cost by the transported quantity since this quantity will be independent from the transportation mode.

At each node to be delivered, three delivery costs will be associated: the cost to be delivered from the depot located near the source either by cryogenic trucks, noted $C_{m}^{c r y o}$, or by gaseous trucks, noted $C_{m}^{g a s}$ and the marginal cost to be delivered by an extra length of pipelines from the previous end of the pipeline system. This marginal cost to supply hydrogen by pipeline to the considered terminal node is calculated with the differences between the 2 average yearly costs

$$
C_{m}^{\text {pipes }}=C_{m 2}-C_{m 1}
$$

with:

- $C_{m 2}$, the equivalent annual costs with the terminal node,

- $C_{m 1}$, the equivalent annual costs without the terminal node.

Finally, we obtain the equivalent annual cost for the trucks by the following calculation:

$$
C_{m}^{\text {trucks }}=\frac{C A P E X_{\text {tractor }}}{\sum_{t=1}^{\text {tractorlifetime }} 1 /(1+i)^{t}}+\frac{C A P E X_{\text {trailer }}}{\sum_{t=1}^{\text {trailerlifetime }} 1 /(1+i)^{t}}+O P E X_{T r}
$$

where trucks is equal to cryo for cryogenic trucks transport and gas for gas trucks transport.

\section{Proposed Approach}

To be consistent over time, we need to find a set of pipes and diameters which satisfies all network constraints each year over the length of the planning horizon. For that purpose, an approach (see Triadou [24]) is to select, among all forecasted scenarios of demand, the maximal one on which the one-period optimization is applied. In most cases, the maximal demand is reached the last year of the 
planning horizon due to the constant growth of demands. This specific year will be called the "target year".

We will consider that the minimal spanning tree and the associated diameters minimizing the costs are provided with the heuristic specified in André et al. [1]. These topologies and sizes will be kept constant throughout the deployment process. Starting from this target year $n p$, a backward process of network deconstruction over time will be performed to justify each additional pipe section to deliver a terminal node called leaf of the minimal spanning tree (knowing that each node is a potential delivery node i.e. no intermediate node without quantity to deliver).

The backward heuristic developed in this approach follows the following steps (See Figure 3):

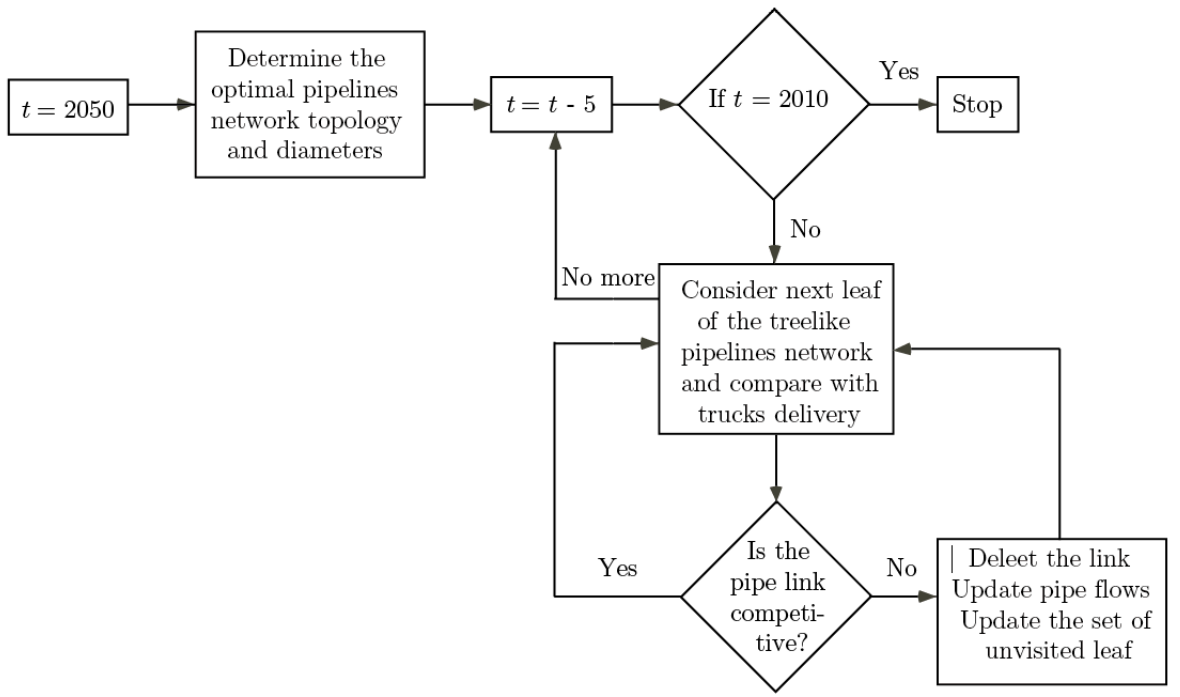

Figure 3: Proposed heuristic

- Initialization: Starting at the final time horizon of the project ( $n p=2050$ in our case), we consider thus that the pipeline network is completely developed using the topology and dimensioning method proposed by André et al.[1]. We obtain a treelike network. This is an important assumption for our heuristic: we assumed that the pipeline network is completely developed, even this option is not competitive with trucks transport.

- Iteration: We consider backward all the preceding time steps, starting from $n p-1$ (= 2045 in our case where we use a time step of 5 years). We determine the initial subset of nodes $S_{l}$ that are the leaves of the initial tree.

- For each terminal leaf of the treelike network belonging to $S_{l}$, we apply the economic criterion presented in the previous subsection to decide if the link by pipe is justified with respect to the trucks delivery 
alternative. If the pipe alternative is economically justified, i.e,

$$
\text { if } C_{m}^{\text {pipes }} \leq \min \left(C_{m}^{\text {cryo }}, C_{m}^{\text {gas }}\right)
$$

jump to the following leaf within the list of terminal nodes. Otherwise, the pipeline truckline is deleted and we must actualize the pipelines flows (which are lower since a part of the final demand is directly delivered by trucks from the hydrogen production station). We add the so created new terminal node to the list of terminal nodes $S_{l}$ to examine in the loop.

- When all terminal leaves belonging to $S_{l}$ are examined, go to the previous time period (of 5 years in our case). The entering pipelines topology for this new application of the terminal leaves examination is the final topology obtained from the subsequent time period.

- Stopping criterion: Proceed backward until the first period of the time horizon is achieved (2010 in our case).

\section{Practical application}

We will test the heuristic proposed in section 4 to the following case study: the deployment of a new hydrogen transmission network for the North of France with one central plant located near Paris. The targeted network will be designed and dimensioned by the heuristic described in André et al. [1]. We obtained a treelike network with the diameters of the pipes corresponding to the minimal investment cost.

\subsection{Demand evolution scenario}

Each delivery node will be associated to a demand level increasing over time between 2010 and 2050 with a time step of 5 years. The market shares prospective relies on the assumptions given by the HyFRance 2 group [11] with a high and a low scenario hydrogen market share for fuels for individual cars (See Table 2).

\begin{tabular}{|l|c|c|c|c|c|c|c|c|c|}
\hline Year & 2010 & 2015 & 2020 & 2025 & 2030 & 2035 & 2040 & 2045 & 2050 \\
\hline High (\%) & 0.1 & 1.5 & 3.3 & 10 & 23.7 & 35 & 55 & 68 & 74.5 \\
\hline Low (\%) & 0.05 & 0.05 & 0.1 & 1 & 2 & 5 & 10 & 15 & 20 \\
\hline
\end{tabular}

Table 2: Demand Scenarios evolutions

\subsection{Observations on the network deployment}

We now detail the main pictures of the network deployment. Figures 4, 5, 6, 7 present the network evolution from 2035 to 2050, North of France zone for the 


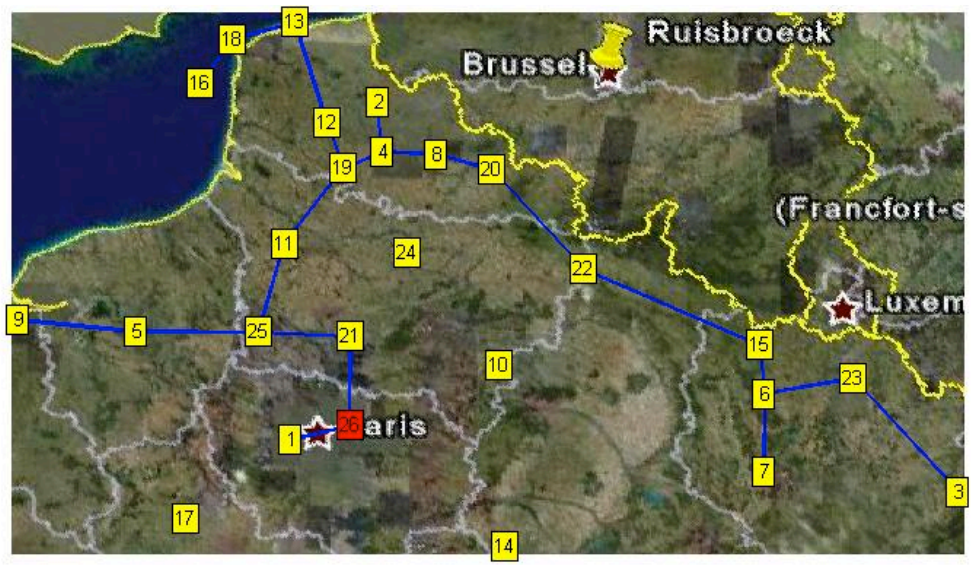

Figure 4: Effective network in 2035, North, High demand

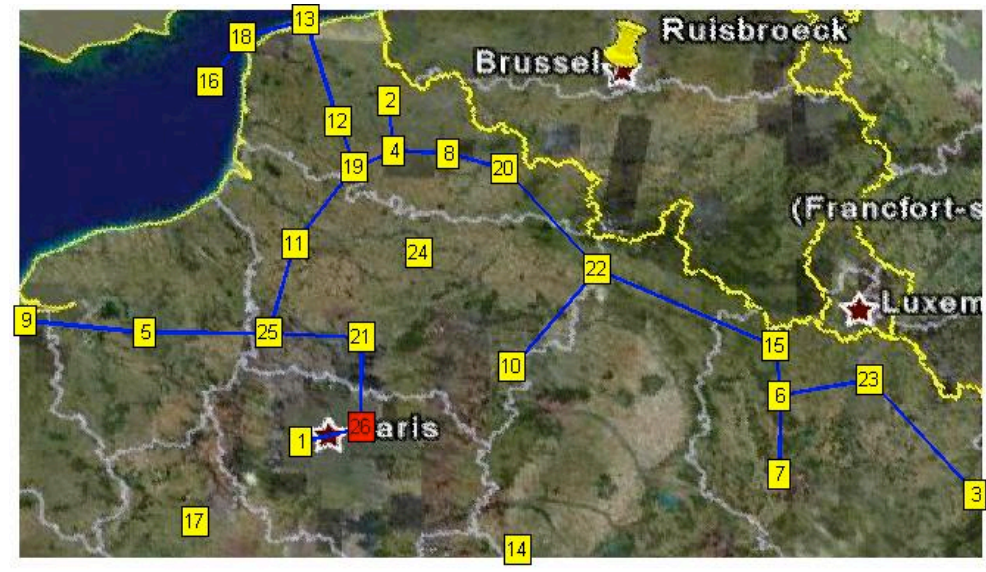

Figure 5: Effective network in 2040, North, High demand

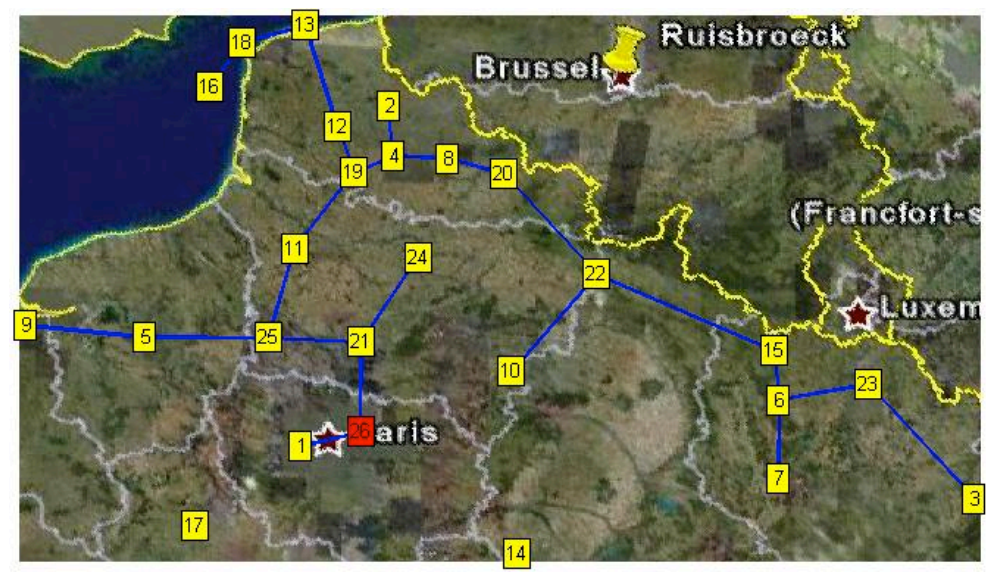

Figure 6: Effective network in 2045, North, High demand 


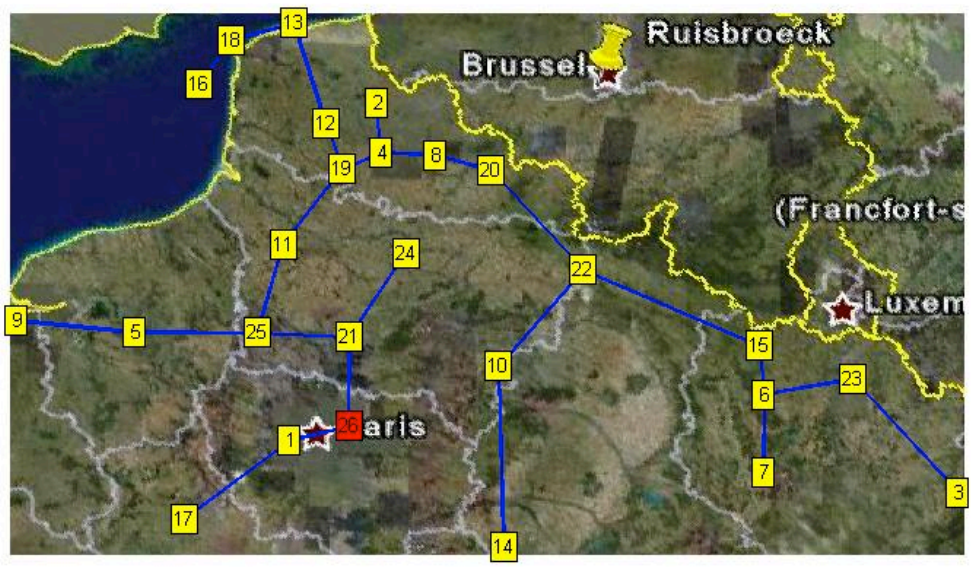

Figure 7: Effective network in 2050, North, High demand

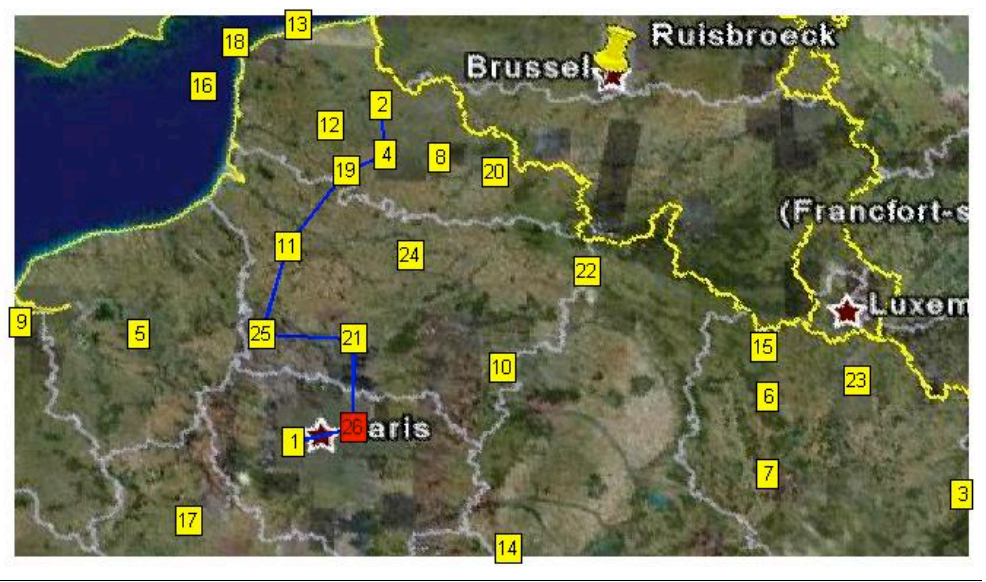

Figure 8: Effective network in 2035, North, Low demand

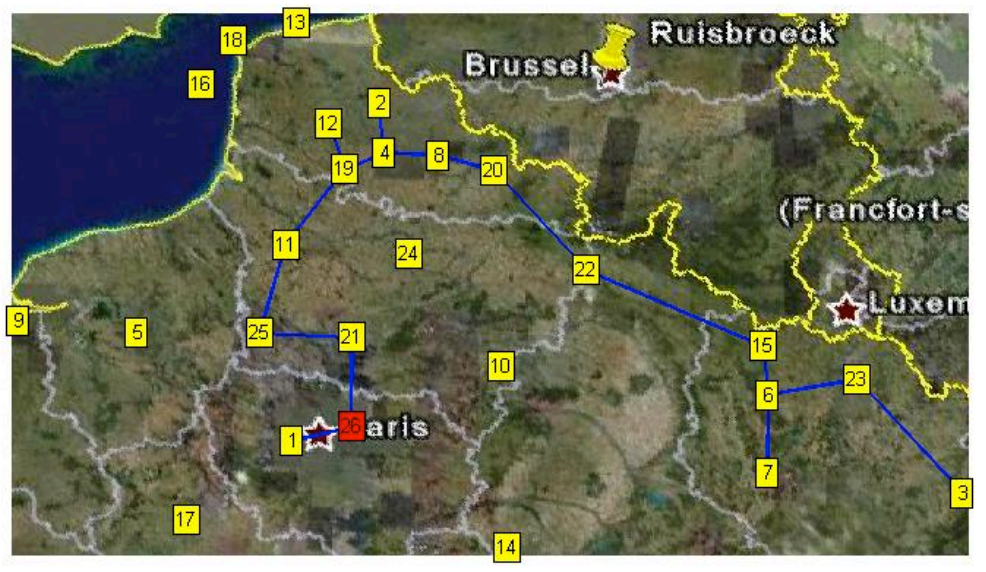

Figure 9: Effective network in 2040, North, Low demand 
high scenario while figures 8, 9, 10, 11 present the network evolution from 2020 to 2050, North of France zone for the low scenario.

These figures show the nodes waiting for a long time before being connected are the closest to the plant. Once connected, they have a direct connection to the plant. The network once started from the central plant moves fast by giving priority to the lines with the highest flows. In fact, once a pipeline has been laid, the marginal costs to reach the following point decreases and there is an advantage to put more flows into the existing pipe. Finally, the network takes a long time to reach some remote points and slowly progresses at the end of the process.

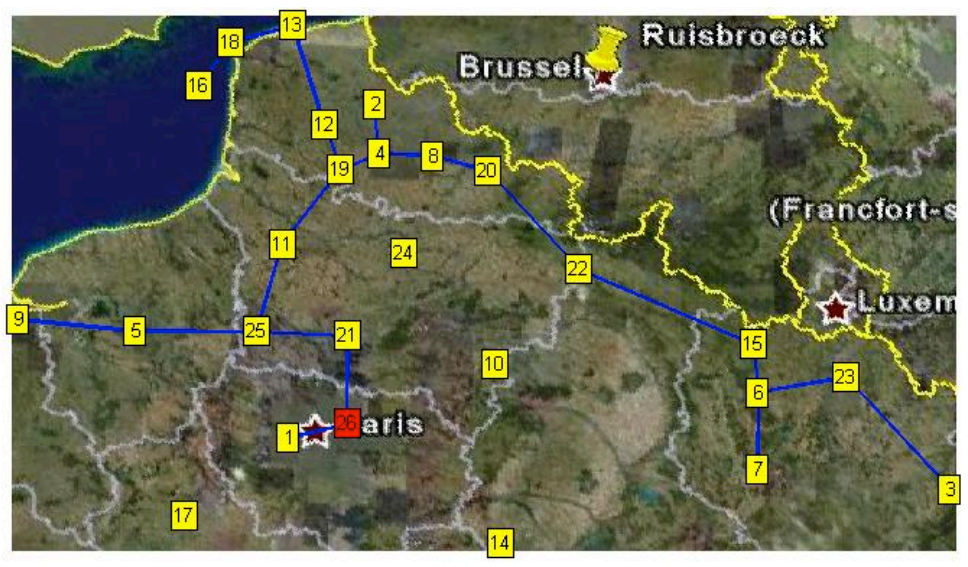

Figure 10: Effective network in 2045, North, Low demand

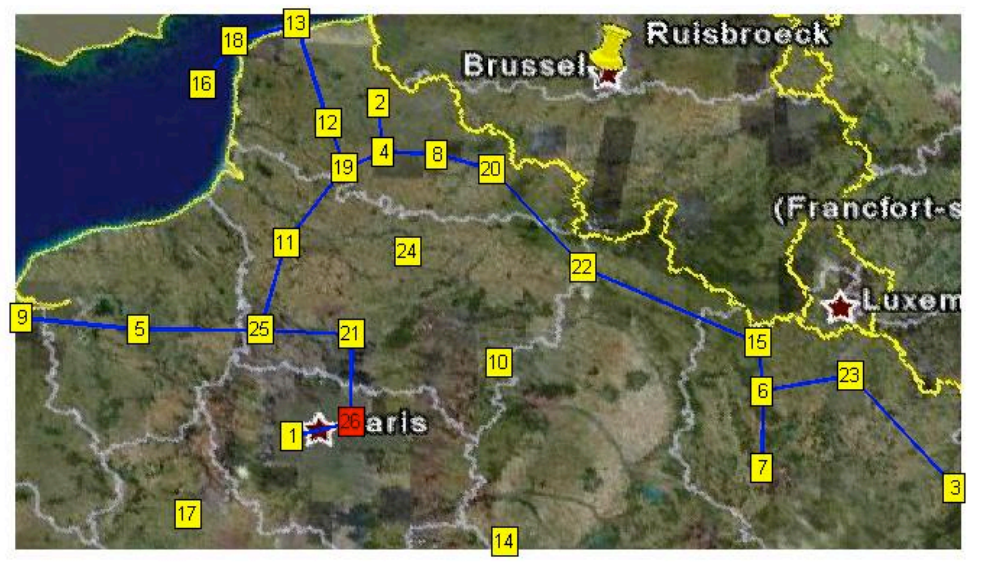

Figure 11: Effective network in 2050, North, Low demand 


\subsection{Observations on the pipeline used to deliver hydrogen}

Figure 12 gives the percentage of the demand delivered by pipeline as a function of time for North of France. This figure shows that for the high scenario, most of the deployment occurs between 2025 and 2030 while for the low assumption, most of the deployment occurs between 2030 and 2040 .

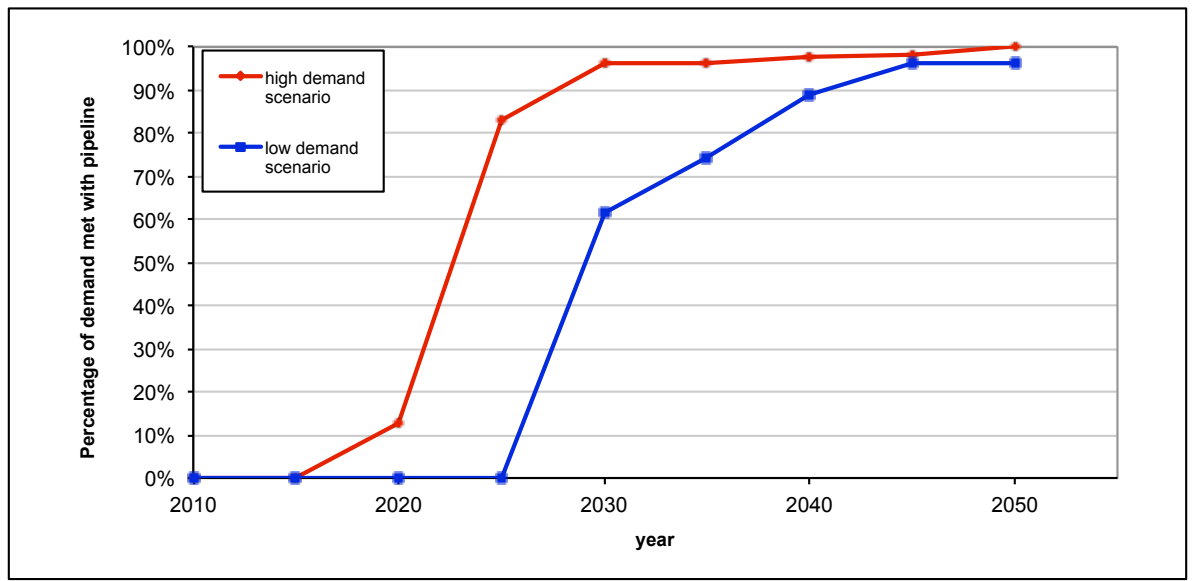

Figure 12: Percentage of demand supplied by pipeline over time, North of France

Figure 13 gives the percentage of the demand delivered by pipeline as a function of the hydrogen market share for North of France. First, we can conclude that as

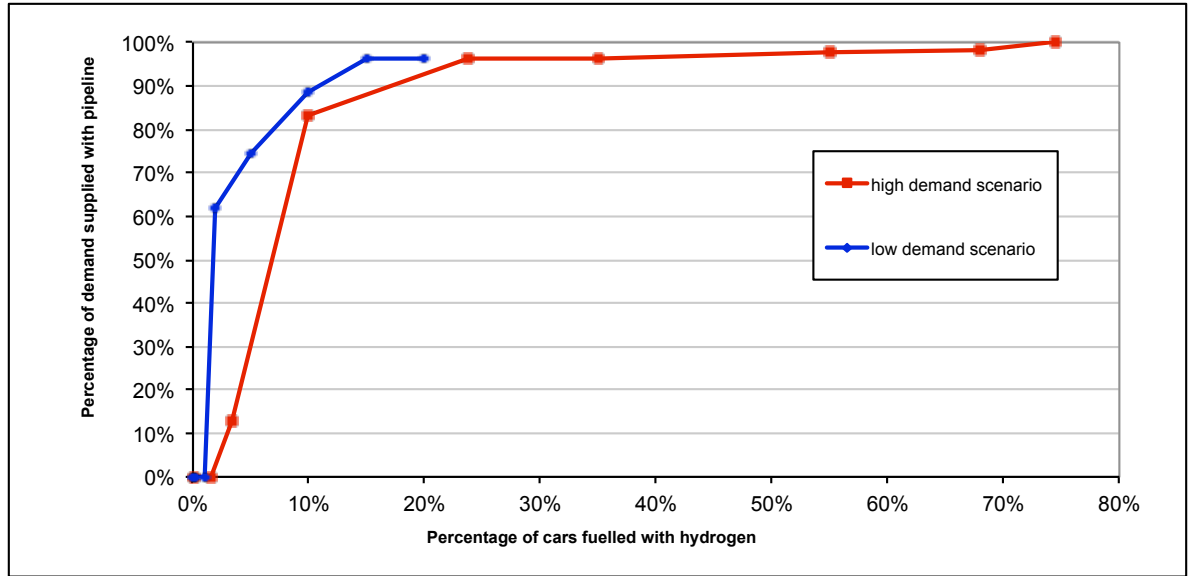

Figure 13: Demand supplied by pipeline as function of hydrogen market share, North of France

soon as a pipeline network is created, it quickly supplies as much as two thirds at least of the total hydrogen demand. This means that the investment made in the pipeline is justified for high level of demand due to economies of scale. Secondly, we see that most of the pipeline network is developed as soon as the market share is worth $10 \%$. 
Note that the "percentage of cars fueled with hydrogen" variable of Figure 13 and the "year" variable of Figure 12 are dependant, the correlation being given by Table 2 .

\subsection{Cost analysis of the different alternatives}

We now answer the following question: what is the cost analysis of the different alternatives? Figure 14 presents the different transportation alternatives used in 2025 in the high scenario for the North of France network. On the graph, the nodes delivered by gaseous trucks are blue circled and the red circled nodes are delivered by liquid trucks.

The costs indicated next to the nodes correspond respectively to the cost by truck or to the average costs of pipelines. These costs include centralized compression costs, liquefaction costs, transport costs and on site compression costs.

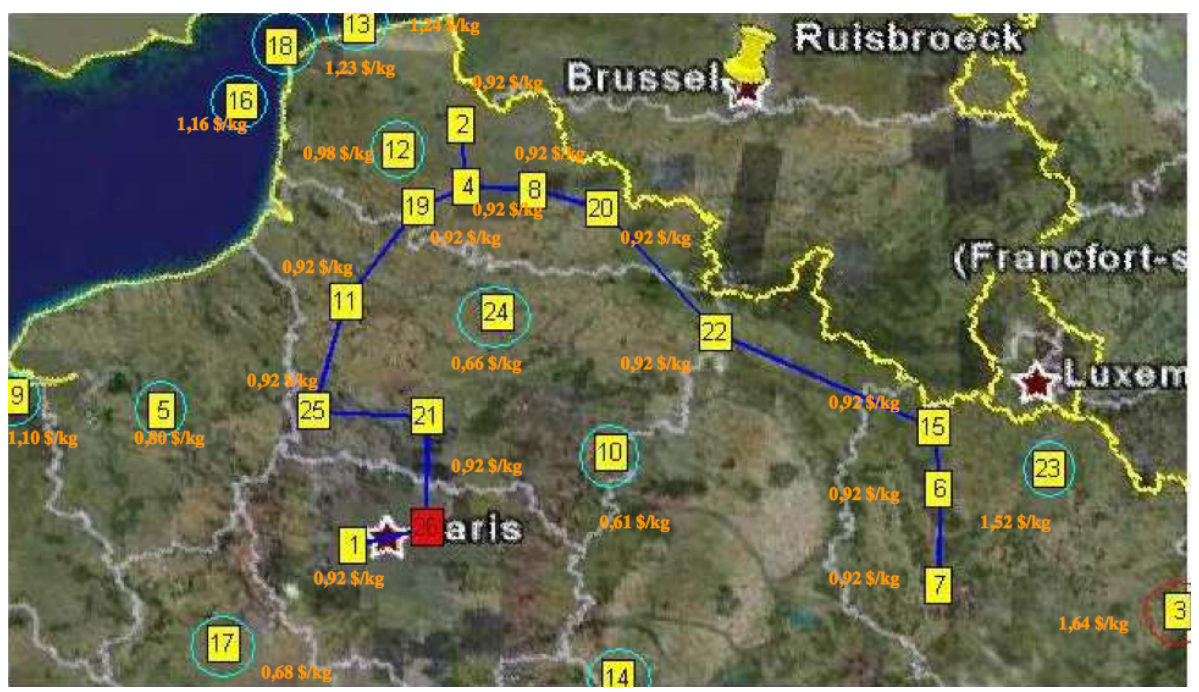

Figure 14: Delivery costs, North of France zone, High demand scenarios, 2025.

We observe naturally that delivery costs increase with the distance to the central plant. In addition, pipeline delivery costs are higher than the delivery by truck for the nearest points to the source and lower than the delivery costs by truck for the furthest points to the source. Indeed, we are averaging the costs of the points connected to the pipeline. As a matter of fact, it demonstrates that the economy of a pipelines network is based on a cost allocation between the close and the remote customers.

Finally, the cryogenic delivery by truck is competitive only at one node (point 3), the most remote location. On all the other nodes, the delivery by gaseous truck is cheaper than the cryogenic option. It means that on this geographic scale, the savings brought by the transport by liquid truck do not offset the extra costs paid by the hydrogen liquefaction. Actually, $300 \mathrm{~km}$ is the minimal distance threshold that can justify the cryogenic option. 


\section{Conclusions and perspectives}

In the context of the scarcity of petroleum products as fuels, hydrogen appears as a promising solution that respects the environment. It is already necessary to define the relevant logistic chain for the transport of hydrogen. In this paper, we put as well in perspective the choice of the pipeline option to transport hydrogen over time compared to the cryogenic and gaseous trucks options. In fact, the specificity of the hydrogen supply chain lies in the fact that the hydrogen may be produced locally and thus, contrary to the case of natural gas coming from long distance, the pipelines compete with other hydrogen carriers: the compressed gas trucks and the liquid cryogenic trucks.

The economic decision criterion used to compare the alternatives is based on the computation of the average yearly cost of the equipment taking into account the initial capital expenditure (CAPEX) and an operational expenditure (OPEX). This yearly average cost was computed for each part of the three different supply modes, including the investment costs, the compression costs, the transportation costs and the liquefaction costs.

We showed that for the short/mid term perspective, i.e. before 2025 and low market share, the trucks are the most economical options. However, for the long term, i.e. beyond 2025, the pipeline option is considered as an economical viable option as soon as the hydrogen energy market share for the car fueling market reaches $10 \%$.

The delivery costs by trucks or pipelines increase with the distance from the central plant but not at the same rate. The pipeline delivery costs are higher than the delivery by truck for the nearest points to the source and lower than the delivery costs by truck for the furthest points to the source. Over short distances gaseous truck delivery will be optimal especially at low flow rates but pipelines may be optimal at higher flow rates. Economies of scale will be visible over long distance pipeline connecting several customers with cheaper marginal costs to reach a new customer from the last served customers by an existing pipeline system.

As possible extension of our work, we plan to consider several sources of hydrogen including not only plants but also hydrogen underground storage. This makes the problem more complicated to solve since it implies to consider first the facility location problem. Another possible extension is to consider other topologies than treelike networks to take into account the network feasibility, that will say to used two-connected networks.

\section{Acknowledgments}

This research was done with the financial support of the National Research Agency ANR (Agence Nationale de la Recherche). The projet research, named ECOTRANSHY for Economy of Transport for Hydrogen, is a project of the research program PAN-H for Programme d'Action Nationale pour l'Hydrogène. 


\section{References}

[1] André J., Auray S., Brac J., De Wolf D., Maisonnier G., Ould Sidi M. and A. Simonet (2013): "Design and Dimensioning of Hydrogen Transmission Pipeline Networks", European Journal of Operational Research, 229, pp. 239251.

[2] Ball M., Wietschel M. and O. Rentz (2007): "Integration of a hydrogen economy into the German energy system: an optimizing modeling approach", International Journal of Hydrogen Energy, 32, pp. 1355-1368.

[3] Balta-Ozkan N. and E. Baldwin (2013): "Spatial development of hydrogen economy in a low-carbon UK energy system", International Journal of Hydrogen Energy, 38, pp. 1209-1224.

[4] Bertsekas D.P. (2000): "Dynamic programming and Optimal Control", Second Edition, Vol. 1. Massachussetts Institute of Technology, Athena Scientific.

[5] Bhaskaran S. and F. Salzborn (1979): "Optimal design of gas pipeline networks", Journal of the operations research society, 30, pp. 1047-1060.

[6] Baufume S., Gruger F., Grube T., Krieg D., Linssen J., Weber M., Hake J.F. and D. Stolten (2013): "GIS-based scenario calculation for a nationwide German hydrogen pipeline infrastructure", International Journal of Hydrogen Energy, 38, pp. 3813-3829.

[7] Brimberg J., Hansen P., Lin K.-W., Mladenovic N. and M. Breton (2003): "An Oil Pipeline Design Problem", Operations Research, 51, pp. 228-239.

[8] Castello P., Tzimas E., Moretto P. and S.D. Paeteves (2005): "Technoeconomic assessment of hydrogen transmission and distribution systems in Europe in the medium and long term", European Commission, Joint Research Center. Report EUR 21586-EN, Luxemburg: Office for Official Publications of the European Communities, Scientific and Technical Research series.

[9] De Wolf D. and Y. Smeers (1996): "Optimal Dimensioning of Pipe Networks with Application to Gas Transmission Networks", Operations Research, 44, pp. 596-608.

[10] US Department of Energy (2006): DOE H2A Delivery Analysis, H2A Delivery Components Model Version 1.1: Users Guide, April 7, 2006, http : //www.hydrogen.energy.gov/h2a elivery.html.

[11] HyFrance 2 project (2007): "Final Report: Evaluation technicoEconomique du développement d'une filière hydrogène en France et de ses impacts sur le système énergétique, l'économie et l'environnement", http : //www.ecolo.org/documents/documents ${ }_{i} n_{f}$ rench/H2 - rapport ${ }_{H}{ }_{H}$ yFrance 0 7.pdf

[12] Johnson N. and J. Ogden (2012): "A spatially-explicit optimization model for long-term hydrogen pipeline planning", International Journal of Hydrogen Energy, 37, pp. 5421-5433. 
[13] Jones T.W., Smith D. (1982): "An Historical Perspective of Net Present Value and Equivalent Annual Cost", Accounting Historians Journal, Volume 9, Number 1.

[14] Katz D.L., Cornel D., Kobayashi R., Poettmann F.H., Vary J.A., Elenbaas J.R., Weinaug C.F., Handbook of Natural Gas Engineering, McGraw-Hill Book Company, Inc., New York, USA, 1959

[15] Kubat P. and J. MaxGregor Smith (2001): "A multi-period network design problem for cellular telecommunication systems", European Journal of Operationnal Research, 134, pp. 439-456.

[16] Lin Z., Fan Y., Ogden J. and D. Sperling (2005): "The Hydrogen Infrastructure Transition Model (HIT) \& Its Application in Optimizing a 50-year Hydrogen Infrastructure for Urban Beijing", UCD-ITS-RR-06-05.

[17] Middleton R.S. and J.M. Bielicki (2009): "A scalable infrastructure model for carbon capture and storage: SimCCS", Energy Policy, 37, pp. 1052-1060.

[18] Mohitpour, Golshan and Murray (2003): Pipeline Design and Construction - A Practical Approach. Second Edition, ASME Press, USA.

[19] Olorunniwo F. O. and P.A. Jensen (1982): "Optimal Capacity Expansion policy for natural Gas transmission networks: A decomposition approach", Engineering Optimization, 6, pp. 13-30.

[20] Parker N., (2005): "Using Natural gas Transmission Pipeline Costs to Estimate Hydrogen Pipeline Costs", Technical report No. UCD-ITS-RR-04-3, Institute of Transportation Studies, University of California, Davis.

[21] Patay E., Ghaffari A., Minoux M. and J. Perrin (2006): "Surrogate approximation for the cost evaluation of infrastructure development for hydrogen as an automotive fuel", Proceedings 19th Mini EURO Conference on the Energy Sector, ORMMES 06, 6-9.

[22] Rothfarb B., Frank H., Rosenbaum D.M., Steiglitz K and D.J. Kleitman (1970): "Optimal design of offshore natural-gas pipeline systems", Operations research, 18, pp. 992-1020.

[23] Smit R., Weeda M. and A. De Groot (2007): "Hydrogen infrastructure development in The Netherlands", International Journal of Hydrogen Energy, 32 , pp. $1387-1395$.

[24] Triadou C. (2003): "Dimensionnement de réseau multipériode et relaxation Lagrangienne", Actes du Congrés ROADEF.

[25] Ting Ze N. (2006): "Optimal Lay-Out of Natural Gas Pipeline Network", 23rd World Gas Conference, Amsterdam.

[26] The National Research Council (2004): The Hydrogen Economy: Opportunities, Costs, Barriers, and R\&D Needs, Appendix E, Spreadsheet data for hydrogen supply Chain analyses

[27] Walters G.A. (1987): "Dynamic programming approach to the optimal design of tree-like networks", Proceedings of the international conference on 
Optimization Techniques and Applications, Singapour, Singapore, pp. 487496.

[28] Yang C. and Ogden J. (2007): "Determining the lowest-cost hydrogen delivery mode", International Journal of Hydrogen Energy, 32, pp. 268-286.

[29] Yi F. (1990): "Capacity planning for a natural gas pipeline in Sweden", Energy Economics, pp. 211-215. 